\title{
Association between homocysteine and white matter hyperintensities in asymptomatic intracranial arterial stenosis: results from a population-based study
}

Hao Yin

Shandong University

Xiang Wang

Shandong University

Yuan-yuan Zhao

Shandong University

Xiao-kang Ji

Shandong University

Shao-wei Sang

Shandong University

Sai Shao

Shandong University

Peng Yan

Shandong University

Shan Li

Shandong University

Yuan Xue

Shandong University

Guang-bin Wang

Shandong University

Ming LV

Shandong University

Fu-zhong Xue

Shandong University

YiFeng Du

Shandong University

Qinjian Sun ( $\nabla$ sqi1210@163.com )

Shandong Provincial Hospital https://orcid.org/0000-0002-9323-9154 


\section{Research article}

Keywords: Homocysteine, White matter hyperintensities, Arteriosclerosis, Asymptomatic intracranial arterial stenosis, Population-based study

Posted Date: October 16th, 2019

DOI: https://doi.org/10.21203/rs.2.16135/v1

License: (c) (i) This work is licensed under a Creative Commons Attribution 4.0 International License.

Read Full License 


\section{Association between homocysteine and white matter hyperintensities in asymptomatic}

intracranial arterial stenosis: results from a population-based study

Hao Yin ${ }^{1}$, Xiang Wang ${ }^{1}$, Yuan-Yuan Zhao ${ }^{1}$, Xiao-Kang Ji², Shao-Wei Sang ${ }^{3}$, Sai Shao ${ }^{4}$, Peng Yan $^{1}$, Shan Li ${ }^{1}$, Yuan Xue ${ }^{1}$, Guang-Bin Wang ${ }^{4}$, Ming Lv ${ }^{3}$, Fu-Zhong Xue ${ }^{2}$, Yi-Feng Du ${ }^{1}$, QinJian $\operatorname{Sun}^{1 *}$

${ }^{1}$ Department of Neurology, Shandong Provincial Hospital Affiliated to Shandong University, Jinan, Shandong, China

${ }^{2}$ Department of Biostatistics, School of Public Health, Shandong University, Jinan, Shandong, China

${ }^{3}$ Department of Clinical Epidemiology, Qilu Hospital affiliated to Shandong University, Jinan, Shandong, China

${ }^{4}$ Department of Radiology, Shandong Medical Imaging Research Institute Affiliated to Shandong University, Jinan, Shandong, China

*Corresponding author:

Qinjian Sun

Department of Neurology, Shandong Provincial Hospital Affiliated to Shandong University, No. 324 Jingwu Weiqi Road, Jinan, Shandong 250021, People’s Republic of China

Tel: +86-(0)53168773276

Email: sqj1210@163.com

Running title: Association between Hcy and WMH in asymptomatic ICAS 


\begin{abstract}
Background: Although homocysteine (Hcy) and white matter hyperintensities (WMH) have been proven to be correlated with increased risks of ischemic stroke, there have been few studies addressing the association between serum Hcy and WMH in a population with asymptomatic intracranial arterial stenosis (aICAS). Thus, the aim of the present study is to describe the association between Hcy and WMH in rural-dwelling Chinese people with aICAS.
\end{abstract}

Methods: In this study, 150 participants diagnosed as aICAS by magnetic resonance angiography were recruited from the Kongcun Town Study, which was a population-based study aimed to investigate the prevalence of aICAS in general population aged 40 to 90 years old, free of ischemic stroke history, and living in the Kongcun town, Pingyin county, Shandong, China. Data on demographics, risk factors, and serum Hcy levels were collected via interview, clinical examination, and laboratory tests. The WMH volumes were calculated through the lesion segmentation tool system for the Statistical Parametric Mapping package based on magnetic resonance imaging. The association between Hcy and WMH volume was analyzed using both linear and logistic regression analysis.

Results: After adjusting for all confounders, high Hcy (HHcy) (serum Hcy $\geq 15$ umol/L) was significantly associated with severe WMH (the highest quartile in WMH volume) (OR: 2.972, 95\%CI: 1.017-7.979, $\mathrm{P}<0.05)$. However, with changing of WMH volumes, only trends towards association with HHcy were observed in all 3 models ( $P$ values only slightly exceeded 0.05$)$. After being stratified by age, sex, or ever smoking, the association between HHcy and WMH became more significant in participants who were $\geq 60$ years old, male, or ever smoker. 
Conclusions: HHcy is associated with severe WMH in rural-dwelling Chinese people with aICAS, especially in participants $\geq 60$ years old, male participants, or ever smokers, indicating these may be risk factors that contribute to the association between HHcy and severe WMH.

Keywords: Homocysteine; White matter hyperintensities; Arteriosclerosis; Asymptomatic intracranial arterial stenosis; Population-based study 


\section{Highlights}

- Hcy levels are associated with WMH in rural-dwelling Chinese people with aICAS.

- Automated, quantitative measurements of WMH volume can avoid inaccuracies.

- Presence of risk factors can increase association between high Hcy and severe WMH. 


\section{Background}

White matter hyperintensities (WMH) are mostly bilateral and symmetrical areas in the white matter that appear hyperintense on proton density and T2-weighted magnetic resonance imaging (MRI) scans [1]. WMH is one of the most important signs of cerebral small vessel disease (CSVD), a group of pathological processes with various aetiologies that affect small arteries, arterioles, venules, and capillaries of the brain [1,2], and is known for its high prevalence in the elderly $[3,4]$. It is associated with increased risks of death, ischemic stroke [5], cognitive decline [6], and dementia [7]. Besides WMH, signs of CSVD include lacunar, recent small subcortical infarcts, enlarged perivascular spaces, microbleeds, and brain atrophy seen with MRI [8].

A series of study found that high neutrophil to lymphocyte ratio and subcutaneous adipose tissue were associated with WMH volumes in a healthy population $[9,10]$. In recent years, an increased number of studies have focused on identifying an association between WMH and homocysteine (Hcy), which is a sulphur-containing amino acid formed during the metabolism of the essential amino acid, methionine. Hcy has also proved to be an independent predictor of stroke risk [11], and a significant risk factor for cognitive decline and dementia [12, 13]. In The Rotterdam Scan Study, Hcy was associated with WMH and silent brain infarcts [14]. In The Northern Manhattan Study, investigators found that elevated Hcy is a risk factor for white matter damage [15]. In patients with symptomatic atherosclerosis disease, higher Hcy levels are also associated with increased WMH volume [16].

However, most of these studies focused on community-based populations of Hispanic, Black, and Caucasian subjects, or patients with symptomatic atherosclerosis disease. Few studies about the association between WMH and Hcy in Chinese people have been conducted, especially in rural-dwelling Chinese people with asymptomatic intracranial arterial stenosis (aICAS). The 
purpose of this study was to investigate the association between Hcy and WMH in rural-dwelling Chinese people with aICAS.

\section{Methods}

\section{Study population}

This study was based on the Kongcun Town Study, which was a population-based study that aimed to investigate the prevalence of aICAS within the general Kongcun town (Pingyin county) population. A total of 2027 participants, who were between the ages of 40 and 90 years and had no prior history of ischemic stroke, were enrolled. Among them, 150 participants were detected arrow intracranial arteries through magnetic resonance angiography (MRA), which consist of bilateral intracranial segments of the internal carotid arteries (ICA), middle cerebral arteries (MCA), posterior cerebral arteries (PCA), anterior cerebral arteries (ACA), intracranial segments of vertebral arteries (VA) and/or the basilar artery (BA). All participants did not experience any symptoms commonly associated with intracranial artery stenosis, such as limb weakness, numbness, aphasia, etc., and did not have a history of stroke, or transient ischemic attacks (TIA) which were rated by a trained investigator. All 150 of these participants were recruited to this present study.

This study was approved by the ethical standards committee on human experimentation at Shandong Provincial Hospital Affiliated to Shandong University. Written informed consent was obtained from all participants. This study was conducted in accordance with the principles for medical research involving human subjects expressed in the Declaration of Helsinki. 


\section{Demographics and risk factors}

Data on age, gender, and smoking or drinking history were obtained through interviews.

Smoking or drinking history were defined as an ever, or never smoking or alcohol consumption.

Overnight fasting, venous blood samples were taken to determine blood glucose levels and obtain lipid profiles from participants which included low density lipoprotein-cholesterol (LDLC), high density lipoprotein-cholesterol (HDL-C), triglyceride (TG), total cholesterol (TC) and

Hcy levels. Height and weight were measured to calculate body mass index (BMI). Hypertension was defined as having either a mean systolic blood pressure $\geq 140 \mathrm{mmHg}$, a mean diastolic blood pressure $\geq 90 \mathrm{mmHg}$, a history of hypertension, or antihypertensive drug use. Diabetes mellitus was defined as having either a history of diabetes mellitus, or a venous blood glucose level $\geq 7.0$ $\mathrm{mmol} / \mathrm{L}$, or using oral antidiabetic drugs or insulin. Finally, hypercholesterolemia was defined as total cholesterol $\geq 6.2 \mathrm{mmol} / \mathrm{L}$.

\section{Hcy measurement}

Overnight fasting, venous blood samples were taken in the morning from all participants. The blood samples were allowed to clot in a $37^{\circ} \mathrm{C}$ water bath for 30 minutes, followed by centrifugation at $3000 \mathrm{rpm}$ for 15 minutes. The serum supernatant was taken and separated into 5 aliquots $\left(200 \mu \mathrm{L}\right.$ each) that were snap frozen in liquid nitrogen before transferring to a $-80{ }^{\circ} \mathrm{C}$ freezer for storage; a method shown to improve stability of Hcy in assays [17]. Blood tests were conducted at the certified clinical laboratory of Shandong Provincial Hospital Affiliated to Shandong University. Participants were placed in either the High Hcy (HHcy) group, defined as having a Hcy level $\geq 15 \mu \mathrm{mol} / \mathrm{L}$, or the normal Hcy group if their Hcy level was less than 15 $\mu \mathrm{mol} / \mathrm{L}$. 


\section{WMH volume measurement}

We calculated the total volume, comprised of both periventricular and deep WMH, in all 150 participants using a LST system which is a lesion segmentation tool for the Statistical Parametric Mapping package (SPM) [18] based on MRI. WMH were considered present if they were visible as hyperintense on proton density and T2-weighted images, without prominent hypointense areas on T1-weighted images to distinguish them from infarctions in white matter. Participants were divided into the following 4 groups based on their total WMH volume: $\leq 0.29 \mathrm{~mL}, 0.30$ to 1.36 $\mathrm{mL}, 1.37$ to $4.28 \mathrm{~mL}$, and $\geq 4.29 \mathrm{~mL}$. The severe $\mathrm{WMH}$ group consisted of patients with $\mathrm{WMH}$ volumes $\geq 4.29 \mathrm{~mL}$.

\section{Data analysis}

The association between HHcy and WMH volume was examined using linear regression analysis. Model 1 adjusted for age and sex, and model 2 additionally adjusted for BMI, hypertension, diabetes mellitus, hypercholesterolemia, HDL-C, LDL-C, and TG. In model 3, ever smoking and drinking were added. Additionally, logistic regression was used to analyse the association between HHcy and severe WMH. Variants adjusted in models 1, 2, and 3 for logistic regression analysis were the same as those adjusted for linear regression. We also evaluated the association between Hcy levels and WMH volume or severe WMH volume using both linear regression analysis and logistic regression analysis after adjusting the same variants.

In order to investigate the association between HHcy and severe WMH volume in participants with different risk factors, we also analysed the association between HHcy and severe WMH volume in participants stratified according to age, sex, and ever smoking. 


\section{Results}

\section{Demographics of study participants}

Of the 150 participants included in this study, 13 participants were excluded due to missing MRI data $(n=3)$ or homocysteine data $(n=10)$. Consequently, the analytical sample included 137 participants with aICAS. The mean age of these participants was 59.9 years (SD \pm 10.1$) ; 40.9 \%$ were men; $78.8 \%$ had hypertension, and $27.0 \%$ had diabetes (Table.1). The mean Hcy level was $15.0 \mu \mathrm{mol} / \mathrm{L}(\mathrm{SD} \pm 6.5)$, while the mean $\mathrm{WMH}$ volume was $3.60 \mathrm{~mL}(\mathrm{SD} \pm 5.60)$ (Table.1). Fig. 1 shows the flowchart of the WMH volume using the LST auto-calculation. The differences were significant between the HHcy $(n=47)$ and the normal Hcy $(n=90)$ groups in age, gender, and ever smoking $(P<0.05)$. Besides, the difference between the HHcy and the normal Hcy groups in WMH volumes was obviously significant $(P<0.01)$ (Table.1).

\section{Association between HCY and WMH}

HHcy levels were significantly associated with severe WMH volumes in model 1 (OR: 3.11, 95 \% CI: 1.239-7.803, $P<0.05$ ), model 2 (OR: 3.013, $95 \%$ CI: 1.139-7.971, $P<0.05$ ) and model 3 (OR: 2.972, $95 \%$ CI: 1.107-7.979, $P<0.05)$ in logistic regression (Table.2). However, there was only a trend towards association between HHcy levels and WMH volumes in all 3 models in linear regression ( $P$ value only slightly exceeded 0.05 in all three models) (Table.2).

\section{Association between HHcy and severe WMH stratified by age, gender or ever smoking}

After being stratified by age, HHcy levels were significantly associated with severe WMH volumes adjusted for all confounders in participants with age $\geq 60$ years (OR:4.266, 95\%CI: 
1.084-16.794, $P<0.05)$. HHcy was also significantly associated with severe WMH in male participants $(P<0.05)$, and participants with ever smoking $(P \leq 0.05)$ (Table.3). However, there was no significant association between HHcy and severe WMH in participants $<60$ years old, female participants, or participants without ever smoking (Table.3).

\section{Discussion}

This study found that HHcy was associated with severe $\mathrm{WMH}$, and adjustments for associated risk factors did not attenuate this effect. The odds of severe WMH were 2.972-fold higher in participants with HHcy compared with those without HHcy. After stratification by different risk factors, HHcy was significantly associated with severe WMH in older participants ( $\geq 60$ years), male participants, and participants with ever smoking. To the best of our knowledge, this is the first study to explore the association between Hcy and WMH in rural-dwelling Chinese people with aICAS.

Previous studies found that there was an association between Hcy and WMH in some community-based studies $[14,15,19]$. In The Rotterdam Scan Study, researchers concluded that Hcy was associated with white matter lesions independent of cardiovascular risk factors, such as the presence of carotid artery plaques, and increased intima-media thickness of the common carotid artery [14]. In the Northern Manhattan Study, Hcy was found to be associated with WMH volume in a population that included Hispanic, Black and Caucasian men [15]. The same conclusion was reached in a study that included 682 participants from the CHA Bundang Medical Center [19]. The results of these studies are consistent with our findings. However, some studies were unable to illustrate the association between Hcy and WMH. In the 
Framingham Offspring Study, investigators could not find an association between plasma Hcy and WMH volume [20]. This inconsistency may arise from their reduced mean Hcy levels, which were $9.8 \mu \mathrm{mol} / \mathrm{L}$ at the fifth offspring examination and $8.3 \mu \mathrm{mol} / \mathrm{L}$ at the seventh examination (versus $15.0 \mu \mathrm{mol} / \mathrm{L}$ in our present study). This might have underestimated the association between Hcy and WMH. In another study that randomly selected individuals from a community in Australia, between the ages of 60 and 64, researchers found that the association was significant only between HHcy and deep WMH, but not periventricular WMH [21]. One possible explanation is that the pathological basis of these two kinds of lesions may be different. Ischemia aetiology is likely to be more important for development of deep WMH, while myelin pallor, or rarefaction, are often related to periventricular WMH [21]. Because we could not calculate volumes of deep or periventricular WMH separately, we were unable to illustrate the association between Hcy with deep or periventricular WMH singularly.

Being this study is a cross-sectional research, it is difficult to conclude a possible causal association between Hcy and WMH. However, a closer look at previously conducted, longitudinal studies suggests contradicting conclusions about this association. To the best of our knowledge, as this present moment, only 4 studies have investigated a longitudinal association between Hcy and WMH, and most of them failed to identify an association between Hcy and WMH $[12,22,23]$. The Epidemiology of Vascular Aging study and the Cardiovascular Health Study are two of the most well-known longitudinal studies [12, 22]. However, considering that only semiquantitative measurements of WMH were used, and sample sizes were small and included only a specific population (only older hypertensive subjects recruited from the Study on Cognition and Prognosis in the Elderly (SCOPE) trial) [23, 24], it is difficult to draw a convincing conclusion about the association between Hcy and WMH. To avoid these 
confounding factors and investigate the association between Hcy and WMH more precisely, a larger, longitudinal study using quantitative measurement of WMH was conducted [25]. In the SMART-MR study, researchers found that elevated Hcy levels were associated with greater progression of $\mathrm{WMH}$, independent of vascular risk factors and severity of atherosclerotic disease [25]. However, what needs to be mentioned is that the SMART-MR study was not a communitybased study. Therefore, larger longitudinal studies, based on general communities are needed to reveal the association between Hcy and WMH progression.

In our current study, HHcy was closely associated with severe WMH, especially in older participants, in male participants, and in participants with ever smoking. The pathological effects of elevated Hcy may explain these results. High levels of Hcy could activate NMDA receptordependent regulation of adherens and tight junctions to increase permeability of the blood-brain barrier [26], which is considered one of the most important pathological factors in CSVD [1]. Furthermore, the activation of NMDA receptors caused by HHcy could also induce neurotoxicity, causing apoptosis of brain cells [27]. Hcy has also been shown to directly induce endothelial damage [3, 28], which has been noted in CSVD [29].

Arterial stiffness is known to accelerate with aging [29]. Men may be less protected than women from this stiffening effect since they have lower circulating levels of oestrogen, a protective factor for cerebral circulation [30]. Smoking could affect endothelial nitric oxide synthase activity which is essential for normal endothelial function [31]. Because of this, the endothelium of brain tissue may be more vulnerable to damage by metabolites and toxicants, such as Hcy. These factors may contribute to the increased WMH seen in elderly people, in male participants, and in smokers with elevated Hcy. 
Because the Hcy level can be regulated by folic acid and vitamin B12, which may be potential therapy targets, it is of great importance to elucidate whether treatment with vitamin supplements can reduce WMH volumes and thus prevent stroke or dementia. Despite the fact that the results of several large trials testing vitamin supplementation failed to show beneficial effects towards preventing vascular diseases [32-34], our study suggests that vitamin supplement trials should focus more on elderly populations, males, and smokers, as they might be more sensitive to damage caused by Hcy.

The strengths of this study are as follows: First, to the best of our knowledge, this is the first study to explore the association between Hcy and WMH in rural-dwelling Chinese people with aICAS. Second, the lesion prediction algorithm implemented using the SPM LST toolbox in this study has been shown to produce reliable, consistent results, and in many cases, is considered a robust gold standard for lesion segmentation [35]. The use of automated, quantitative measurements of WMH avoided pitfalls of previously used semi-quantitative methods that could invariably result in inaccurate measurements of $\mathrm{WMH}$, and researcher bias. However, there are several limitations in the present study as well. First, because we did not calculate volumes of deep or periventricular WMH separately, we were unable to illustrate the individual associations between Hcy and deep, or periventricular, WMH. Larger longitudinal studies focusing on the association between Hcy and deep or periventricular WMH are needed to help understand the potential different roles of Hcy in deep WMH versus periventricular WMH. Second, as a crosssectional study, it is impossible to conclude a possible causal association between Hcy and WMH. Third, Hcy levels were not corrected for vitamin B12 and folic acid levels in this study, and since they are involved in the metabolism of Hcy, they could have lowered blood plasma Hcy levels in participants [36]. 


\title{
Conclusions
}

In conclusion, this study found that HHcy is associated with severe WMH in rural-dwelling Chinese people with aICAS, especially in participants $\geq 60$ years old, male participants, and participants with ever smoking, indicating these may be risk factors that contribute to the association between HHcy and severe WMH.

\begin{abstract}
Abbreviations
ACA, anterior cerebral arteries; aICAS, asymptomatic intracranial arterial stenosis; BA, basilar artery; BMI, body mass index; CSVD, cerebral small vessel disease; Hcy, homocysteine; HDL-C, high density lipoprotein-cholesterol; HHcy, High Hcy; LDL-C, low density lipoproteincholesterol; MCA, middle cerebral arteries; MRA, magnetic resonance angiography; MRI, magnetic resonance imaging; PCA, posterior cerebral arteries; ICA, internal carotid arteries; SCOPE, Study on Cognition and Prognosis in the Elderly; SPM, statistical parametric mapping package; TC, total cholesterol; TG, triglyceride; TIA, transient ischemic attacks; VA, vertebral arteries; WMH, White matter hyperintensities.
\end{abstract}

\section{Acknowledgements}

We would like to thank all the study participants, staff of the participating hospitals, and the Steering Committee Members of this study.

\section{Authors' contributions}

QJS, YFD and FZX conceived and designed the research. HY, WX, YYZ, XKJ, SWS, SS, PY, SL and YX acquired the data. WX, YYZ, SS, PY, SL, YX and GBW analyzed and interpreted the data. HY and WX draft the manuscript. ML, FZX, YFD and QJS made critical revisions of the manuscript. All authors approved the final manuscript.

\section{Funding}

This study was supported by the grants from Jinan Science and Technology Bureau (grants no. 201503003 and 201704101) and Department of Science and Technology of Shandong Province (grants no. 2014GSF118106, 2016GSF201062, and ZR2017MH114). Q Sun received grants from the National Natural Science Foundation of China (NSFC, grant no. 81971128). Y Du received grants from the National Major Science and Technology Projects of China (grant no. 2017YFC1310100) and the National Natural Science Foundation of China (NSFC, grant no. 
8171101298). F Xue received grants from International Science and Technology Cooperation Major Project of Ministry of Science and Technology (grant no. 2014DFA 32830). M Lv received grants from the National Major Science and Technology Projects of China (grant no. 2017YFC0907003).

\section{Availability of data and materials}

The data that support the findings of this study are available from the corresponding author upon reasonable request.

\section{Ethics approval and consent to participate}

This study was approved by the ethical standards committee on human experimentation at Shandong Provincial Hospital Affiliated to Shandong University.

\section{Consent for publication}

Not applicable.

\section{Competing Interest}

The authors declare that they have no competing interests. 


\section{References}

1. Pantoni L. Cerebral small vessel disease: from pathogenesis and clinical characteristics to therapeutic challenges. The Lancet Neurology. 2010, 9(7):689-701.

2. Staszewski J, Piusinska-Macoch R, Brodacki B, Skrobowska E, Stepien A. IL-6, PF-4, $\mathrm{sCD} 40 \mathrm{~L}$, and homocysteine are associated with the radiological progression of cerebral small-vessel disease: a 2-year follow-up study. Clinical interventions in aging. 2018, 13:1135-1141.

3. Cloonan L, Fitzpatrick KM, Kanakis AS, Furie KL, Rosand J, Rost NS. Metabolic determinants of white matter hyperintensity burden in patients with ischemic stroke. Atherosclerosis. 2015, 240(1):149-153.

4. Ryu WS, Woo SH, Schellingerhout D, Jang MU, Park KJ, Hong KS, Jeong SW, Na JY, Cho KH, Kim JT et al. Stroke outcomes are worse with larger leukoaraiosis volumes. Brain: a journal of neurology. 2017, 140(1):158-170.

5. Conijn MM, Kloppenborg RP, Algra A, Mali WP, Kappelle LJ, Vincken KL, van der Graaf Y, Geerlings MI, Group SS. Cerebral small vessel disease and risk of death, ischemic stroke, and cardiac complications in patients with atherosclerotic disease: the Second Manifestations of ARTerial disease-Magnetic Resonance (SMART-MR) study. Stroke. 2011, 42(11):3105-3109.

6. Silbert LC, Nelson C, Howieson DB, Moore MM, Kaye JA. Impact of white matter hyperintensity volume progression on rate of cognitive and motor decline. Neurology. 2008, 71(2):108-113.

7. Kester MI, Goos JD, Teunissen CE, Benedictus MR, Bouwman FH, Wattjes MP, Barkhof F, Scheltens P, van der Flier WM. Associations between cerebral small-vessel disease and Alzheimer disease pathology as measured by cerebrospinal fluid biomarkers. JAMA neurology. 2014, 71(7):855-862.

8. Wardlaw JM, Smith EE, Biessels GJ, Cordonnier C, Fazekas F, Frayne R, Lindley RI, O'Brien JT, Barkhof F, Benavente OR et al. Neuroimaging standards for research into small vessel disease and its contribution to ageing and neurodegeneration. The Lancet Neurology. 2013, 12(8):822-838.

9. Nam KW, Kwon HM, Jeong HY, Park JH, Kim SH, Jeong SM, Yoo TG, Kim S. High neutrophil to lymphocyte ratio is associated with white matter hyperintensity in a healthy population. Journal of the neurological sciences. 2017, 380:128-131.

10. Nam KW, Kwon H, Kwon HM, Park JH, Jeong HY, Kim SH, Jeong SM, Kim HJ, Hwang SS. Abdominal fatness and cerebral white matter hyperintensity. Journal of the neurological sciences. 2019, 404:52-57.

11. Homocysteine Studies C. Homocysteine and risk of ischemic heart disease and stroke: a meta-analysis. Jama. 2002, 288(16):2015-2022.

12. Dufouil C, Alperovitch A, Ducros V, Tzourio C. Homocysteine, white matter hyperintensities, and cognition in healthy elderly people. Annals of neurology. 2003, 53(2):214-221.

13. Ravaglia G, Forti P, Maioli F, Martelli M, Servadei L, Brunetti N, Porcellini E, Licastro F. Homocysteine and folate as risk factors for dementia and Alzheimer disease. The American journal of clinical nutrition. 2005, 82(3):636-643. 
14. Vermeer SE, van Dijk EJ, Koudstaal PJ, Oudkerk M, Hofman A, Clarke R, Breteler MM. Homocysteine, silent brain infarcts, and white matter lesions: The Rotterdam Scan Study. Annals of neurology. 2002, 51(3):285-289.

15. Wright CB, Paik MC, Brown TR, Stabler SP, Allen RH, Sacco RL, DeCarli C. Total homocysteine is associated with white matter hyperintensity volume: the Northern Manhattan Study. Stroke. 2005, 36(6):1207-1211.

16. Kloppenborg RP, Nederkoorn PJ, van der Graaf Y, Geerlings MI. Homocysteine and cerebral small vessel disease in patients with symptomatic atherosclerotic disease. The SMART-MR study. Atherosclerosis. 2011, 216(2):461-466.

17. Ueland PM, Refsum H, Stabler SP, Malinow MR, Andersson A, Allen RH. Total homocysteine in plasma or serum: methods and clinical applications. Clinical chemistry. 1993, 39(9):1764-1779.

18. Schmidt P, Gaser C, Arsic M, Buck D, Forschler A, Berthele A, Hoshi M, Ilg R, Schmid VJ, Zimmer $\mathrm{C}$ et al. An automated tool for detection of FLAIR-hyperintense white-matter lesions in Multiple Sclerosis. NeuroImage. 2012, 59(4):3774-3783.

19. Park SY, An SA, Lee HB, Kim Y, Kim NK, Kim SH, Kim OJ, Oh SH. Different impact of hyperhomocysteinemia on cerebral small vessel ischemia and cervico-cerebral atherosclerosis in non-stroke individuals. Thrombosis research. 2013, 131(1):e12-16.

20. Seshadri S, Wolf PA, Beiser AS, Selhub J, Au R, Jacques PF, Yoshita M, Rosenberg IH, D'Agostino RB, DeCarli C. Association of plasma total homocysteine levels with subclinical brain injury: cerebral volumes, white matter hyperintensity, and silent brain infarcts at volumetric magnetic resonance imaging in the Framingham Offspring Study. Archives of neurology. 2008, 65(5):642-649.

21. Sachdev P, Parslow R, Salonikas C, Lux O, Wen W, Kumar R, Naidoo D, Christensen H, Jorm A. Homocysteine and the brain in midadult life: evidence for an increased risk of leukoaraiosis in men. Archives of neurology. 2004, 61(9):1369-1376.

22. Longstreth WT, Jr., Katz R, Olson J, Bernick C, Carr JJ, Malinow MR, Hess DL, Cushman M, Schwartz SM. Plasma total homocysteine levels and cranial magnetic resonance imaging findings in elderly persons: the Cardiovascular Health Study. Archives of neurology. 2004, 61(1):67-72.

23. Narayan SK, Firbank MJ, Saxby BK, Stansby G, Hansrani M, O'Brien JT, Ford GA. Elevated plasma homocysteine is associated with increased brain atrophy rates in older subjects with mild hypertension. Dementia and geriatric cognitive disorders. 2011, 31(5):341-348.

24. Saxby BK, Harrington F, Wesnes KA, McKeith IG, Ford GA. Candesartan and cognitive decline in older patients with hypertension: a substudy of the SCOPE trial. Neurology. 2008, 70(19 Pt 2):1858-1866.

25. Kloppenborg RP, Geerlings MI, Visseren FL, Mali WP, Vermeulen M, van der Graaf Y, Nederkoorn PJ, Group SS. Homocysteine and progression of generalized small-vessel disease: the SMART-MR Study. Neurology. 2014, 82(9):777-783.

26. Beard RS, Jr., Reynolds JJ, Bearden SE. Hyperhomocysteinemia increases permeability of the blood-brain barrier by NMDA receptor-dependent regulation of adherens and tight junctions. Blood. 2011, 118(7):2007-2014.

27. Lipton SA, Kim WK, Choi YB, Kumar S, D'Emilia DM, Rayudu PV, Arnelle DR, Stamler JS. Neurotoxicity associated with dual actions of homocysteine at the N-methyl- 
D-aspartate receptor. Proceedings of the National Academy of Sciences of the United States of America. 1997, 94(11):5923-5928.

28. Poggesi A, Pasi M, Pescini F, Pantoni L, Inzitari D. Circulating biologic markers of endothelial dysfunction in cerebral small vessel disease: A review. Journal of cerebral blood flow and metabolism: official journal of the International Society of Cerebral Blood Flow and Metabolism. 2016, 36(1):72-94.

29. Faßbender K, Mielke O, Bertsch T, Nafe B, Fröschen S, Hennerici M. Homocysteine in cerebral macroangiography and microangiopathy. The Lancet. 1999, 353(9164):15861587.

30. Haast RA, Gustafson DR, Kiliaan AJ: Sex differences in stroke. Journal of cerebral blood flow and metabolism: official journal of the International Society of Cerebral Blood Flow and Metabolism. 2012, 32(12):2100-2107.

31. Burke A, Fitzgerald GA. Oxidative stress and smoking-induced vascular injury. Progress in cardiovascular diseases. 2003, 46(1):79-90.

32. Toole JF, Malinow MR, Chambless LE, Spence JD, Pettigrew LC, Howard VJ, Sides EG, Wang $\mathrm{CH}$, Stampfer M. Lowering homocysteine in patients with ischemic stroke to prevent recurrent stroke, myocardial infarction, and death: the Vitamin Intervention for Stroke Prevention (VISP) randomized controlled trial. Jama. 2004, 291(5):565-575.

33. Bonaa KH, Njolstad I, Ueland PM, Schirmer H, Tverdal A, Steigen T, Wang H, Nordrehaug JE, Arnesen E, Rasmussen K et al. Homocysteine lowering and cardiovascular events after acute myocardial infarction. The New England journal of medicine. 2006, 354(15):1578-1588.

34. Group VTS. B vitamins in patients with recent transient ischaemic attack or stroke in the VITAmins TO Prevent Stroke (VITATOPS) trial: a randomised, double-blind, parallel, placebo-controlled trial. The Lancet Neurology. 2010, 9(9):855-865.

35. Guerrero R, Qin C, Oktay O, Bowles C, Chen L, Joules R, Wolz R, Valdes-Hernandez MC, Dickie DA, Wardlaw J et al. White matter hyperintensity and stroke lesion segmentation and differentiation using convolutional neural networks. NeuroImage Clinical. 2018, 17:918-934.

36. Spence JD. B vitamin therapy for homocysteine: renal function and vitamin B12 determine cardiovascular outcomes. Clinical chemistry and laboratory medicine. 2013, 51(3):633-637. 


\section{Figures}

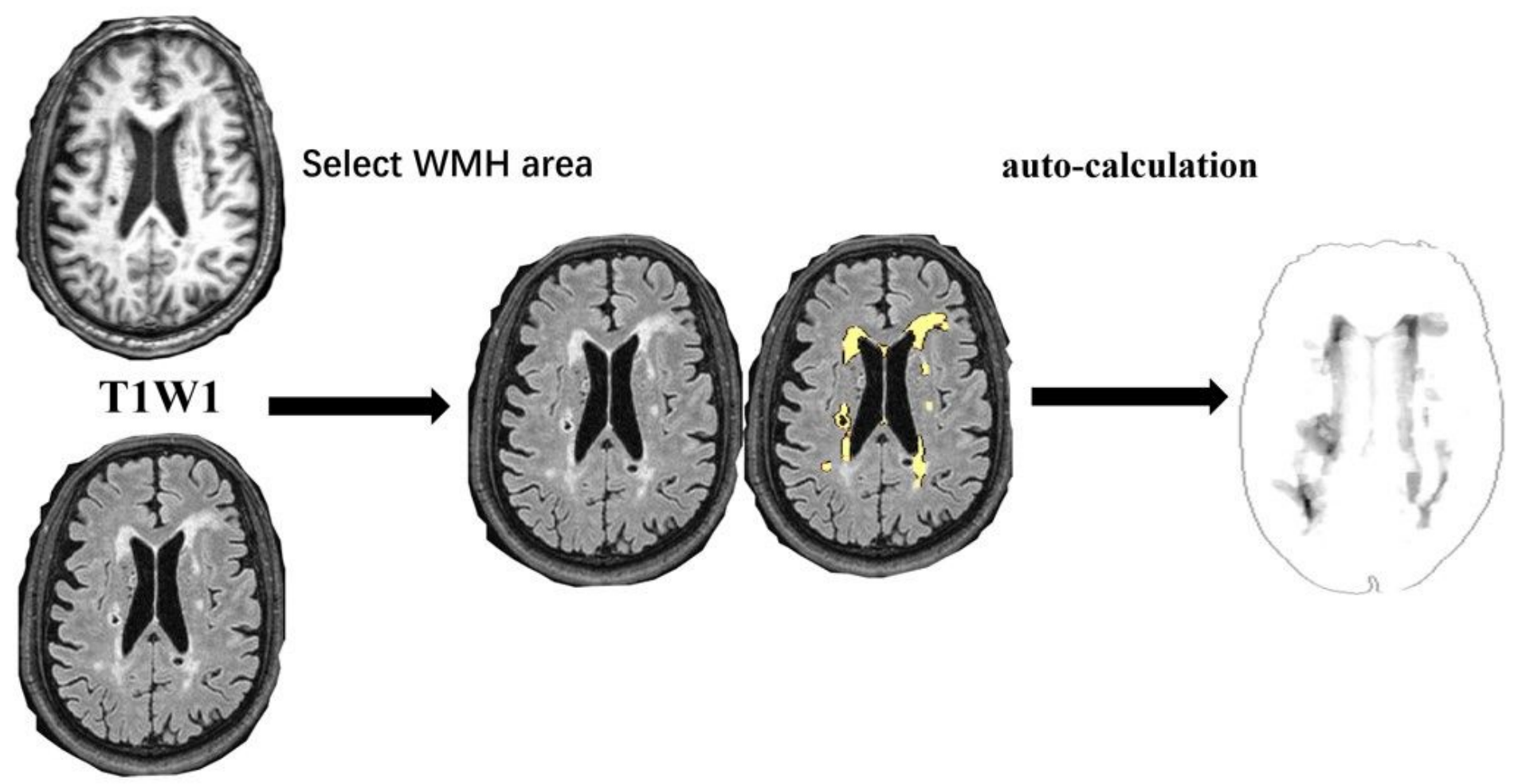

\section{Flair}

Figure 1

flowchart of the WMH volume using the LST auto-calculation

\section{Supplementary Files}

This is a list of supplementary files associated with this preprint. Click to download.

- Table1.pdf

- Table2.pdf

- Table3.pdf 